\title{
Realignment of a Surgical Gastric Sleeve Using the Apollo Endoscopic Suturing Platform in a Patient With Postoperative Persistent Nausea and Vomiting
}

\author{
Alsadiq Al Hillan a, d, Mujtaba Mohamed ${ }^{\mathrm{a}}$, Kadhim Al-Banaa ${ }^{\mathrm{b}}$, Diana Curras-Martin ${ }^{\mathrm{a}}$, \\ Steven Gorceyc, Mohammad Hossain ${ }^{\mathrm{a}}$
}

\begin{abstract}
Laparoscopic sleeve gastrectomy is a restrictive procedure without the malabsorptive component of gastric bypass or duodenal switch. Reported common complications include bleeding, stenosis, misalignment, staple line leaks, and severe regurgitate symptoms. This case represents an uncommon complication characterized by gastroesophageal junction outflow obstruction due to redundant tissue from post-surgical change in the fundus. Repair with complete resolution of symptoms was achieved by performing endoscopy gastroplasty with the Apollo overstitch suturing system.
\end{abstract}

Keywords: Post sleeve gastrectomy; Laparoscopy; Endoscopic suturing/gastroplasty

\section{Introduction}

Sleeve gastrectomy is a bariatric procedure in which the majority of the greater curvature of the stomach is removed creating a tubular stomach. Generally reserved for patients with body mass index $(\mathrm{BMI})>60 \mathrm{~kg} / \mathrm{m}^{2}$ [1], in 2016 , it was the most commonly performed bariatric procedure in the world. Reported complication includes bleeding, stenosis, gastric leaks, acute angulations in the sleeve segment, and sever reflux disease [2-5]. We present a patient who underwent gastric sleeve surgery and presented with persistent nausea and vomiting secondary to gastroesophageal junction obstruction by redundant fundal tissue which was repaired by

Manuscript submitted June 12, 2019, accepted June 20, 2019

aDepartment of Medicine, Jersey Shore University Medical Center, Hackensack Meridian Health, Neptune, NJ 07753, USA

bepartment of Medicine, Beaumont Hospital-Dearborn, 18101 Oakwood Blvd Dearborn, MI 48124, USA

'Department of Gastroenterology, Jersey Shore University Medical Center, Hackensack Meridian Health, Neptune, NJ 07753, USA

${ }^{\mathrm{d} C}$ Corresponding Author: Alsadiq Alhillan, Department of Medicine, Jersey Shore University Medical Center, Hackensack Meridian Health, Neptune, NJ 07753, USA. Email: alsadiq.alhillan@gmail.com

doi: https://doi.org/10.14740/jcs380 endoscopic suturing technique with complete resolution of symptoms.

\section{Case Report}

This is a 58-year-old obese woman with no past medical history who underwent surgical sleeve gastrectomy and hiatal hernia repair in 2016. After few months of procedure, she presented with complaints of post-meal epigastric pain, nausea, vomiting and intermittent dysphagia. She denied constipation or abdominal distension. Vitals signs, physical examination and laboratory findings were all unremarkable. Upper gastrointestinal series showed delay of contrast from the esophagus into the proximal sleeve. The patient underwent an upper endoscopy which showed compromise at the entrance of the sleeve caused by a redundant fold of tissue extending from the fundus to the lesser curvature direction, creating a ball valve effect (Fig. 1). This was presumed to be result from the compromised esophageal emptying. There was no evidence of stricture and the remainder of the sleeve was straight with an appropriate diameter. The antrum and duodenum were normal. Treatment options were discussed, including both surgical and endoscopic management. As pa-

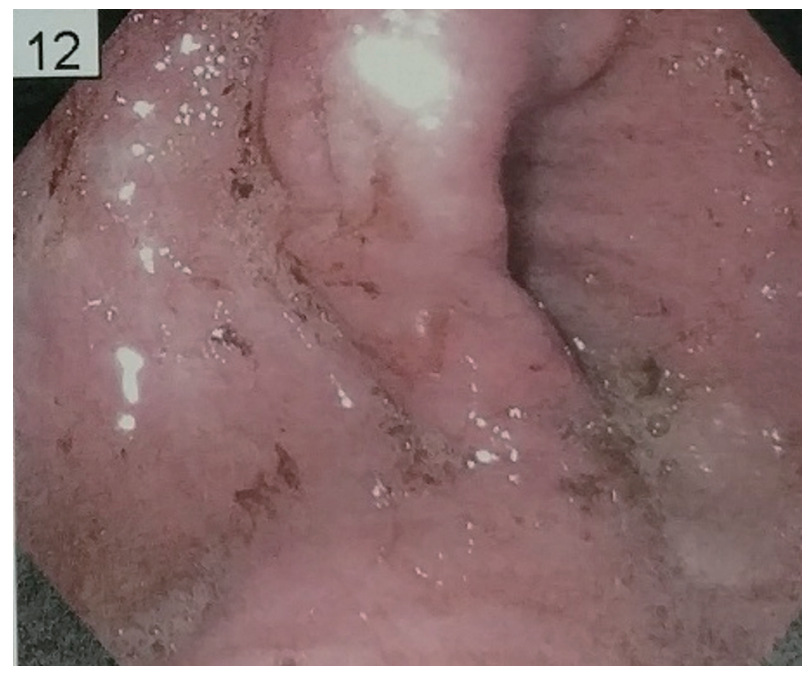

Figure 1. Gastroesophageal junction obstruction by redundant tissue. 


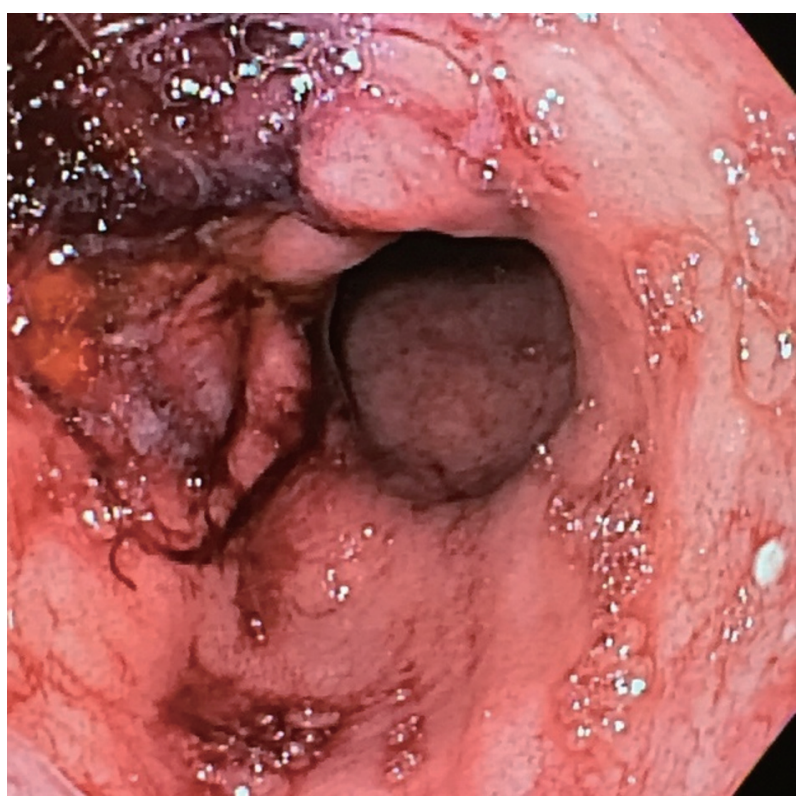

Figure 2. Post endoscopic suture repair showing intact gastroesophageal junction.

tient agreed the decision was made to perform endoscopic intervention using the Apollo overstitch suturing device. Two running 2.0 propylene non-absorbable sutures were utilized to "tack back" the redundant fold into to fundus proper. The technique involved using a helical grasper to grab the tissue on the lesser curve side first which was blocking the sleeve entrance and then suturing it to greater curve side of the retained fundus. Four passages were made with each suture, cinching each at the end. Afterwards, endoscopy showed a patent gastroesophageal junction (GEJ) to sleeve corridor with complete resolution of the obstruction (Fig. 2). The patient tolerated the procedure very well, was discharged on the same day, and started on a low residue diet. After 2 weeks of follow-up visit, the patient reported complete resolution of the symptoms. Postoperative upper gastrointestinal series showed no further delay of sleeve filling.

\section{Discussion}

Laparoscopic sleeve gastrectomy is a restrictive procedure without the malabsorptive component of gastric bypass or duodenal switch. It involves resection of two-thirds of the stomach to provide increased satiety and decreased appetite. Gastric sleeve was the most commonly performed bariatric surgery for weight loss in the USA in 2016 [6]. Reported common complications include bleeding, stenosis, misalignment, staple line leaks, and severe regurgitant symptoms [2-5]. This case represents an uncommon complication characterized by gastroesophageal junction outflow obstruction due to redundant tissue from post-surgical change in the fundus. The patient presented with persistent nausea, vomiting as well as intermittent dysphagia. Repair with complete resolution of symptoms was achieved by performing endoscopy gastroplasty with the
Apollo overstitch suturing system.

Overstitch endoscopic suturing system demonstrated to be feasible, secure and effective in this field endoluminal closure of the gastrointestinal tract wall. Our case is an example to support the progress of the emerging field of endoluminal surgery. Current systems can replicate traditional surgical suturing patterns in the endoluminal environment. Available data include a study that proved the safety and efficacy of Apollo overstitch endoscopic suturing system in augmentation of gastroesophageal junction for the treatment of gastroesophageal reflux disease (GERD) [7, 8].

We recommend that any patient reporting severe upper gastrointestinal symptoms after a gastric sleeve procedure should be evaluated for this condition, and if found, endoscopic suturing can be strongly considered, as revision surgery carries significant morbidity.

\section{Conclusions}

This case represents an uncommon complication characterized by gastroesophageal junction outflow obstruction due to redundant tissue from post-surgical change in the fundus. Repair with complete resolution of symptoms was achieved by performing endoscopic gastroplasty with the Apollo overstitch suturing system; endoscopic suturing can be strongly considered, as revision surgery carries significant morbidity.

\section{Acknowledgments}

We would like to express our deepest appreciation to all those who provided us with the possibility to complete this case report. A special faculty member Dr. Hossain, with contribution in stimulating suggestions and encouragement, helped us to coordinate our project especially in writing this case report.

\section{Financial Disclosure}

None.

\section{Conflict of Interest}

None.

\section{Informed Consent}

The patient described in the case report had given informed consent for the case report to be published.

\section{Author Contributions}

AA contributed to study design/planning, data collection/entry, preparation of manuscript and literature analysis/search; 
DCM contributed to preparation of manuscript and literature analysis/search; MH, KAB, MM and SG contributed to data interpretation and preparation of manuscript.

\section{References}

1. Giordano S, Victorzon M. Bariatric surgery in elderly patients: a systematic review. Clin Interv Aging. 2015;10:1627-1635.

2. Lalor PF, Tucker ON, Szomstein S, Rosenthal RJ. Complications after laparoscopic sleeve gastrectomy. Surg Obes Relat Dis. 2008;4(1):33-38.

3. Dapri G, Cadiere GB, Himpens J. Laparoscopic seromyotomy for long stenosis after sleeve gastrectomy with or without duodenal switch. Obes Surg. 2009;19(4):495499.

4. Keidar A, Appelbaum L, Schweiger C, Elazary R, Bal- tasar A. Dilated upper sleeve can be associated with severe postoperative gastroesophageal dysmotility and reflux. Obes Surg. 2010;20(2):140-147.

5. Marcotte E, Chand B. Management and prevention of surgical and nutritional complications after bariatric surgery. Surg Clin North Am. 2016;96(4):843-856.

6. Clinical Issue committee of the American Society for Metabolic and Bariatric Surgery. Sleeve gastrectomy as a bariatric procedure. Surg Obes Relat Dis. 2007;3(6):573576.

7. Han J, Chin M, Fortinsky KJ, Sharaiha R, Gostout CJ, Chang KJ. Endoscopic augmentation of gastroesophageal junction using a full-thickness endoscopic suturing device. Endosc Int Open. 2018;6(9):E1120-E1125.

8. Halvax P, Diana M, Nagao Y, Marescaux J, Swanstrom L. Experimental evaluation of the optimal suture pattern with a flexible endoscopic suturing system. Surg Innov. 2017;24(3):201-204. 